\title{
THE KNASTER-TARSKI THEOREM VERSUS MONOTONE NONEXPANSIVE MAPPINGS
}

\author{
RAFAEL ESPÍNOLA AND ANDRZEJ WIŚNICKI
}

\begin{abstract}
Let $X$ be a partially ordered set with the property that each family of order intervals of the form $[a, b],[a, \rightarrow)$ with the finite intersection property has a nonempty intersection. We show that every directed subset of $X$ has a supremum. Then we apply the above result to prove that if $X$ is a topological space with a partial order $\preceq$ for which the order intervals are compact, $\mathcal{F}$ a nonempty commutative family of monotone maps from $X$ into $X$ and there exists $c \in X$ such that $c \preceq T c$ for every $T \in \mathcal{F}$, then the set of common fixed points of $\mathcal{F}$ is nonempty and has a maximal element. The result, specialized to the case of Banach spaces gives a general fixed point theorem that drops almost all assumptions from the recent results in this area. An application to the theory of integral equations of Urysohn's type is also given.
\end{abstract}

\section{INTRODUCTION}

M. R. Alfuraidan and M. A. Khamsi asked in [1] whether the classical fixed point theorems for nonexpansive mappings still hold for monotonenonexpansive mappings. An interplay between the order and metrical structure of the space turned out to be very fruitful in the years that followed, including the Bishop-Phelps technique [11], counterparts of Banach's contraction principle (see, e.g., [13, 12, 10, 9]) and numerous applications, from linear and nonlinear matrix equations, differential and integral equations, to game theory. For a recent account of the theory we refer the reader to [4.

Let $(M, \preceq)$ be a set with a partial order. A mapping $T: M \rightarrow M$ is said to be monotone (or increasing) if $T(x) \preceq T(y)$ whenever $x \preceq y$. If in addition $(M, d)$ is a metric space then $T$ is said to be monotone nonexpansive if

$$
d(T(x), T(y)) \leq d(x, y)
$$

for every comparable $x, y \in M$ (i.e., $x \preceq y$ or $y \preceq x$ ). Fixed point theory for nonexpansive mappings is broad and it is natural to study its counterpart for monotone nonexpansive maps. It was initiated quite recently in [2]. In [3], an analogue of the Browder-Göhde fixed point theorem in uniformly convex spaces was obtained. The next step might be to attack a counterpart of classical Kirk's theorem in Banach spaces with weak normal structure,

2010 Mathematics Subject Classification. Primary 06A45, 54F05; Secondary: 34A12, 46B20.

Key words and phrases. monotone mapping; nonexpansive mapping; fixed point; partially ordered set; directed set; Banach space; Urysohn-type equation. 
and then to look for some counterexamples of Alspach's type (for a background of metric fixed point theory, [6] is recommended). Then we could examine a vast number of more or less interesting generalizations of nonexpansive mappings from the order-theoretical point of view that have been introduced for over fifty years now (metric fixed point theory seems to be a real phenomenon here). Such works have recently started to appear.

The aim of this note is to show that fixed point theory for monotone nonexpansive-type mappings is not that rich. For its existential part, it appears to be an application of the classical Knaster-Tarski theorem (also known as the Abian-Brown theorem), see e.g., [7, Theorem 2.1.1], and its generalization to commutative family of monotone mappings. We show in Section 2 a general fixed point theorem with an independent proof that drops almost all assumptions from the recent results in this area, see Corollary 1 and the comments after it. A counterpart of Kirk's theorem follows as a very special case. In Section 3 we apply our results to the theory of functional integral equations of Urysohn's type.

\section{Fixed point theorems}

The following lemma is crucial for our work. In this generality it is probably new but some special cases follow for example from [4, Prop. 2.32], combined with [8, Prop. 1.1.7]. Let $X$ be a set with a partial order $\preceq$. Consider the sets $[a, \rightarrow)=\{x \in X: a \preceq x\}$ and $(\leftarrow, b]=\{x \in X: x \preceq b\}$. Along this work the concept of order intervals in $X$ will be restricted to the sets $[a, \rightarrow)$ and $[a, b]=[a, \rightarrow) \cap(\leftarrow, b]$.

Remember that a subset $J$ of a partially ordered set $X$ is directed if each finite subset of $J$ has an upper bound in $J$.

Lemma 1. Let $X$ be a partially ordered set for which each family of order intervals of the form $[a, b],[a, \rightarrow)$ with the finite intersection property has a nonempty intersection. Then every directed subset of $X$ has a supremum.

Proof. Let $J$ be a directed subset of $X$ and define

$$
K=\bigcap_{x \in J}[x, \rightarrow) .
$$

For each $n$ and a sequence $\left\{x_{1}, \ldots, x_{n}\right\} \subseteq J$ an upper bound in $J$ of the sequence is in $\bigcap_{i=1}^{n}\left[x_{i}, \rightarrow\right)$ and so, from the finite intersection property, $K$ is nonempty. Take now $\left\{z_{1}, \ldots, z_{n}\right\} \subseteq K$ and consider $\bigcap_{i=1}^{n}\left[x_{i}, z_{i}\right]$ which is again nonempty because the same upper bound as above is still in this intersection. Therefore the family $\{[x, z]: x \in J, z \in K\}$ has the finite intersection property too and $K_{0}=\bigcap_{x \in J, z \in K}[x, z]$ is nonempty. Moreover, it is clear that $K_{0} \subseteq K$ and each element of $K_{0}$ is a lower bound of $K$. Hence $K_{0}$ is a singleton and it is, in fact, the supremum of $J$.

Remark 1. Notice that Lemma 1 holds for topological partially ordered spaces for which order intervals are compact.

If we combine Lemma 1 and the Knaster-Tarski theorem we obtain immediately, 
Theorem 1. Let $X$ be a topological space with a partial order $\preceq$ for which order intervals are compact and let $T: X \rightarrow X$ be monotone. If there exists $c \in X$ such that $c \preceq T(c)$, then the set of all fixed points of $T$ is nonempty and has a maximal element.

But the application of Lemma 1 is wider. Having it we obtain a short and independent proof of the following strengthening of Theorem 1 .

Theorem 2. Let $X$ be a topological space with a partial order $\preceq$ for which order intervals are compact and let $\mathcal{F}$ be a nonempty commutative family of monotone maps from $X$ into $X$. If there exists $c \in X$ such that $c \preceq T c$ for every $T \in \mathcal{F}$, then the set of common fixed points of $\mathcal{F}$ is nonempty and has a maximal element.

Proof. Let

$$
J_{0}=\left\{x \in X: x=T_{1} T_{2} \ldots T_{n} c: T_{1}, \ldots, T_{n} \in \mathcal{F}, n \in \mathbb{N}\right\} \cup\{c\} .
$$

It is not difficult to see that $J_{0}$ is a directed set, $x \preceq T x$ and $T x \in J_{0}$ for each $x \in J_{0}$ and $T \in \mathcal{F}$. Furthermore, if $\mathcal{J}$ is a chain of directed subsets of $X$ containing $J_{0}$ with the above properties, then $\bigcup \mathcal{J}$ is a directed set with these properties too, i.e., $x \preceq T x$ and $T x \in \bigcup \mathcal{J}$ for each $x \in \bigcup \mathcal{J}$ and $T \in \mathcal{F}$. By Kuratowski-Zorn's lemma there exists a maximal directed set $J \subseteq X$ which contains $J_{0}$ with these properties and let $s=\sup J$ whose existence follows from Lemma 1. Then $x \preceq s$ and hence $x \preceq T x \preceq T s$ for every $x \in J$ and $T \in \mathcal{F}$. Thus each $T s$ is an upper bound for $J$ and consequently $s \preceq T s$ for $T \in \mathcal{F}$. It follows from the maximality of $J$ that $s=T s \in J$ for each $T \in \mathcal{F}$ and clearly $s$ is a maximal fixed point of $\mathcal{F}$.

Remark 2. Theorem 2 can be also deduced by combining Lemma 1 with DeMarr's theorem [5, Theorem 1].

We are thus led to the following corollary.

Corollary 1. Let $X$ be a Banach space with a partial order $\preceq$ and let $\tau$ be a Hausdorff topology on $X$ such that the order intervals are $\tau$-closed. Suppose $C$ is a (nonempty) $\tau$-compact subset of $X$ and $\mathcal{F}$ a (nonempty) commutative family of monotone maps from $C$ into $C$. Then $\mathcal{F}$ has a common fixed point if and only if there exists $c \in C$ such that $c \preceq f(c)$ for every $f \in \mathcal{F}$. Moreover, the set of common fixed points of $\mathcal{F}$ has a maximal element.

Let us list the improvements of Corollary 1 over known results till date:

- We do not impose any conditions on a Banach space $X$.

- We consider an arbitrary Hausdorff topology $\tau$ while in most papers the classical weak topology is considered or at least a topology for which a Banach space $X$ satisfies a rather strong geometrical assumption (the $\tau$-Opial condition).

- A subset $C$ of $X$ is $\tau$-compact and in general need be neither convex nor bounded.

- We assume that $T: C \rightarrow C$ is monotone only and need be neither monotone-nonexpansive nor continuous. 
- Rather than considering a single mapping $T$, we consider any family of commuting monotone mappings.

- And finally, apart from the existential result we obtain a qualitative information about the set of fixed points that is sometimes helpful in applications.

We conclude this section with a few special cases of Corollary 1 .

Theorem 3 (see [2, Theorem 2.1]). Let X be a Banach space. Let $\tau$ be a topology on $X$ such that $X$ satisfies the $\tau$-Opial condition. Let $\preceq$ be a partial order on $X$ such that order intervals are convex and $\tau$-closed. Let $C$ be a bounded convex $\tau$-compact nonempty subset of $X$ and let $T: C \rightarrow C$ be a monotone nonexpansive mapping. Assume that there exists $x_{0} \in C$ such that $x_{0}$ and $T\left(x_{0}\right)$ are comparable. Then $T$ has a fixed point.

Theorem 4 ([3, Theorem 4.1]). Let $(X,\|\cdot\|, \preceq)$ be a partially ordered Banach space such that order intervals are closed and convex. Assume $X$ is uniformly convex in every direction. Let $C$ be a nonempty weakly compact convex subset of $X$ and let $T: C \rightarrow C$ be a monotone nonexpansive mapping. Assume there exists $x_{0} \in C$ such that $x_{0}$ and $T\left(x_{0}\right)$ are comparable. Then $T$ has a fixed point.

Theorem 5 ([1, Theorem 3.3]). Let $(X,\|\cdot\|, \preceq)$ be a partially ordered Banach space for which order intervals are convex and closed. Assume $X$ is uniformly convex. Let $C$ be a nonempty convex closed bounded subset of $X$ and let $T: C \rightarrow C$ be a continuous monotone asymptotically nonexpansive mapping. Then $T$ has a fixed point if and only if there exists $x_{0} \in C$ such that $x_{0}$ and $T\left(x_{0}\right)$ are comparable.

Theorem 6 ([1, Theorem 3.6]). Let $(X,\|\cdot\|, \preceq)$ be a partially ordered Banach space for which order intervals are convex and closed. Assume $X$ is uniformly convex space that satisfies the monotone weak-Opial condition. Let $C$ be a nonempty convex closed bounded subset of $X$ and let $T: C \rightarrow C$ be monotone asymptotically nonexpansive mapping. Then $T$ has a fixed point if and only if there exists $x_{0} \in C$ such that $x_{0}$ and $T\left(x_{0}\right)$ are comparable.

\section{EXAMPLES OF APPLiCATION}

Weak conditions assumed in the results obtained in the previous section allow us to weaken conditions on examples of applications of monotonicity results. One of these examples is provided, for instance, by [2, Section 3].

Let $\Omega$ be a measure space, and consider the integral equation

$$
x(t)=g(t)+\int_{\Omega} F(t, s, x(s)) d \mu(s), \quad t \in \Omega,
$$

where

i) $g \in L^{2}(\Omega$,$) ,$

ii) $F: \Omega \times \Omega \times L^{2}(\Omega,) \rightarrow$ is measurable and monotone in its third coordinate. 
iii) There exists a non-negative function $h(\cdot, \cdot) \in L^{2}(\Omega \times \Omega)$ and $M \in$ $[0,1 / 2)$ such that

$$
|F(t, s, x)| \leq h(t, s)+M|x|,
$$

where $t, s \in \Omega$ and $x \in L^{2}(\Omega$,$) .$

As it is shown in [2, Section 3], we can associate to this integral equation the operator $J: L^{2}(\Omega,) \rightarrow L^{2}(\Omega$,$) given by$

$$
(J y)(t)=g(t)+\int_{\Omega} \tilde{F}(t)(y)(s) d \mu(s)
$$

where $\tilde{F}(t)(y)(s)=F(t, s, y(s))$. Then it can be shown, in the same terms as in [2], that $J$ sends the whole $L^{2}(\Omega$,$) to a closed ball of sufficiently large$ radius. Now, if we consider the weak topology as $\tau$ in Theorem 2 it can be shown that order intervals are closed with respect to this topology. Finally we need to guarantee that there exists a $c \in L^{2}(\Omega$,$) which is comparable$ with $J(c)$. This will be furnished by the extra condition in the next theorem.

Theorem 7. Under the above assumptions, we have that:

i) The integral equation (3.1) has a non-negative solution provided we assume that $g(t)+\int_{\Omega} F(t, s, 0) d \mu(s) \geq 0$ for almost every $t \in \Omega$.

ii) The integral equation (3.1) has a non-positive solution provided we assume that $g(t)+\int_{\Omega} F(t, s, 0) d \mu(s) \leq 0$ for almost every $t \in \Omega$.

Proof. Notice that the added condition implies that $J(0) \geq 0$ in i) and $J(0) \leq 0$ in ii). The conclusion follows then after Theorem 1 .

Remark 3. Comparing this example with the one provided in [2, Section

$3]$ we can notice that no nonexpansive condition is imposed on $F$ and this allows us to weaken the conditions on the measure space which is no longer required to be finite. In fact, our approach does not even require the measure space to be $\sigma$-finite as it is the case for some other close examples to this one in the literature as the one developed in [4, Section 7.2.2].

\section{ACKnowledgements}

R. Espínola has been partially supported by DGES (MTM2015-65242-C21-P). This work was developed while A. Wiśnicki was visiting the University of Seville in the spring of 2017. He wishes to thank the Department of Mathematical Analysis and IMUS (Instituto de Investigaciones Matemáticas de la Universidad de Sevilla) for hospitality. The authors would like to thank the referee and, especially, the Editor Professor Stanisław Kwapień for insightful comments on the manuscript, providing us with an elegant proof of Theorem 2 and suggestions for the preparation of the final version of this work.

\section{REFERENCES}

[1] M. R. Alfuraidan and M. A. Khamsi, A fixed point theorem for monotone asymptotically nonexpansive mappings, Proc. Amer. Math. Soc., to appear.

[2] M. Bachar and M. A. Khamsi, Fixed points of monotone mappings and application to integral equations, Fixed Point Theory Appl. (2015), 2015:110, 7 pp. 
[3] B. A. Bin Dehaish and M. A. Khamsi, Browder and Göhde fixed point theorem for monotone nonexpansive mappings, Fixed Point Theory Appl. 2016, 2016:20, 9 pp.

[4] S. Carl and S. Heikkilä, Fixed point theory in ordered sets and applications. From differential and integral equations to game theory, Springer, New York, 2011.

[5] R. DeMarr, Common fixed points for isotone mappings, Colloq. Math. 13 (1964), $45-48$.

[6] K. Goebel, W. A. Kirk, Topics in metric fixed point theory, Cambridge University Press, Cambridge, 1990.

[7] A. Granas, J. Dugundji, Fixed point theory, Springer-Verlag, New York, 2003.

[8] S. Heikkilä and V. Lakshmikantham, Monotone iterative techniques for discontinuous nonlinear differential equations, Marcel Dekker, Inc., New York, 1994.

[9] J. Jachymski, The contraction principle for mappings on a metric space with a graph, Proc. Amer. Math. Soc. 136 (2008), 1359-1373.

[10] J. J. Nieto and R. Rodríguez-López, Contractive mapping theorems in partially ordered sets and applications to ordinary differential equations, Order 22 (2005), 223-239 (2006)

[11] R. R. Phelps, Support cones in Banach spaces and their applications, Advances in Math. 13 (1974), 1-19.

[12] A. C. M. Ran, M. C. B. Reurings, A fixed point theorem in partially ordered sets and some applications to matrix equations, Proc. Amer. Math. Soc. 132 (2004), $1435-1443$.

[13] M. Turinici, Fixed points for monotone iteratively local contractions, Demonstratio Math. 19 (1986), 171-180.

Departamento de Análisis Matemático - imus, Universidad de Sevilla, Apdo. de Correos 1160, 41080 Sevilla, Spain

E-mail address: espinola@us.es

Department of Mathematics, Rzeszów University of Technology, Al. Powstańców Warszawy 12, 35-959 Rzeszów, Poland

E-mail address: awisnicki@prz.edu.pl 